\title{
Prolonged Distribution of Tranilast in the Eyes after Topical Application onto Eyelid Skin
}

\author{
Gerard Lee See, ${ }^{a, b}$ Florencio Arce Jr., ${ }^{a, b}$ Shoko Itakura, ${ }^{a}$ Hiroaki Todo, ${ }^{a}$ and Kenji Sugibayashi*, ${ }^{*}$ \\ ${ }^{a}$ Graduate School of Pharmacy and Pharmaceutical Sciences, Josai University; 1-1 Keyakidai, Sakado, Saitama \\ 350-0295, Japan: and ${ }^{b}$ Department of Pharmacy, University of San Carlos; Nasipit, Talamban, Cebu 6000, the \\ Philippines.
}

Received March 28, 2020; accepted April 28, 2020

Tranilast, a lipophilic drug with various ophthalmic applications, was used as a model drug to establish the possibility of delivering lipophilic drugs through the eyelid skin. Pharmacokinetics and tissue distribution studies were conducted employing three application methods (topical application onto eyelid skin, eye drops, and intravenous injection in rats) to broaden the significance of delivering drugs through the eyelids. A two-compartment open model analysis was used for intravenous route while a non-compartmental evaluation was used for topical applications to estimate the pharmacokinetic parameters. Eyelid skin application, eye drops, and intravenous administration had mean residence times (MRTs) of 8.07, 1.79, and $3.25 \mathrm{~h}$ in the eyeball and 10.8, 1.29, and $2.97 \mathrm{~h}$ in the conjunctiva, correspondingly. In the eyeball, topical application of tranilast onto the eyelids corresponded to a 4.5- and 2.5-fold higher MRT compared with eye drops and intravenous administration, respectively. An 8.4- or 3.6-fold higher MRT was observed in the conjunctiva after topical application compared with eye drops or intravenous administration, respectively. This indicated a gradual penetration of tranilast into the eyeball and conjunctiva, subsequently a slow elimination from these target tissues.

Key words pharmacokinetics; tranilast; skin; drug delivery; residence time

\section{Introduction}

Eye drops remained to be the preferred method of delivering ophthalmic drugs owing to accessibility and ease of application. However, anatomical and physiological complexities hinder the controlled delivery and bioavailability of drugs given as eye drops. ${ }^{1)}$

During recent years, the eyelid skin, site where topical application of drugs are possible, has been given attention as an option to eye drops in the therapy of recurring ocular conditions. Several works have validated the capacity of the eyelids to support the delivery of drugs into the ocular tissues. ${ }^{2-5)}$ Our previous work showed that topical application of drugs onto the eyelids yielded high absorption in the conjunctiva and the eyeball, which was mainly be attributed to the thin skin barrier, the stratum corneum, and lower amounts of neutral lipids in the eyelid skin. ${ }^{5}$

Tranilast, anthranilic acid, is a lipophilic drug which have wide applications against ocular conditions such as allergic conjunctivitis, ${ }^{6)}$ prevention of post-operative adhesion after strabismus surgery ${ }^{7)}$ treatment of corneal allergic inflammation, ${ }^{8)}$ and prevention of fibrous opacification of the posterior lens capsule after cataract extraction ${ }^{9)}$ among others. Although tranilast is currently available as an eye drop on the market, only insignificant amounts of the administered drug penetrates to reach the intraocular tissue targets. Repeated application of eye drops in recurring ocular conditions may lead to unwanted systemic exposure and patient inconvenience. To the best of our knowledge, this is the first study to demonstrate the capacity of the eyelids in delivering a lipophilic model drug to ocular target tissues.

In the present study, tranilast, a lipophilic drug with various ophthalmic applications, was used as the model compound to establish the possibility of delivering lipophilic drugs from the eyelid skin into the eyeball. Pharmacokinetics and tissue distribution studies were implemented using tranilast solution administered in three methods (intravenous administration, topical application onto the eyelid skin, and eye drops) to further explore the significance of delivering drugs through the eyelids.

\section{Experimental}

Materials Tranilast was procured from Tokyo Chemical Industry Co., Ltd. (Tokyo, Japan). Tranilast solution (0.1\%) was prepared by dissolving the chemical in sufficient amounts of phosphate-buffered saline (PBS; pH 7.4).

Pharmacokinetics and Tissue Distribution Studies in Rats The experimental procedure was approved by the Institutional Animal Care and Use Committee of Josai University (approval number: JU 18-003), and the study was conducted in conformity with the Association for Research in Vision and Ophthalmology (ARVO) statement for the use of animals. All possible efforts were made to reduce the quantity of animals used in the investigation.

Hairless rats (WBM/ILA-Ht, male, 8 weeks of age, body weight of $200 \pm 5 \mathrm{~g}$ ) were obtained from Ishikawa Experimental Animal Laboratories (Fukaya, Saitama, Japan). The animals were housed appropriately in a controlled environment (12 h light-dark cycle, $25^{\circ} \mathrm{C}, 45 \%$ relative humidity) with free access to food and water. All rats were intraperitoneally anesthetized with three types of anesthesia $(0.38 \mathrm{mg} / \mathrm{kg}$ medetomidine, $2.5 \mathrm{mg} / \mathrm{kg}$ butorphanol, and $2 \mathrm{mg} / \mathrm{kg}$ midazolam) prior to experiments. Rats were ethically sacrificed using pentobarbital sodium $\left(100 \mathrm{mg} / \mathrm{kg}\right.$, intraperitoneally (i.p.)). ${ }^{5,10)}$

Forty-five rats were individually weighed and arbitrarily 
assigned to one of the three groups (intravenous, eye drops, and topical eyelid). Table 1 provides details of the study design. Rats were anesthetized and maintained in prone position throughout the experiment. The intravenous group was administered with tranilast solution via its tail vein whereas the eye drops group was instilled with tranilast solution directly into the eyes by means of a micropipette. A Teflon ${ }^{\mathrm{TM}}$ tube $(2 \mathrm{~cm}$ in length and $0.48 \mathrm{~cm}$ in diameter) was set on the lower eyelids using cyanoacrylate bond and then loaded with tranilast solution using a micropipette for topical eyelid application. The concentration of tranilast in the eyelid skin samples from the group administered with eyedrops was not determined because it is not the intended target tissue for eye drop administration. Obtaining such samples are clinically irrelevant because the drug is directed into the eyeball and the eyelid skin will not serve as a reservoir in the case of eye drop administration.

Blood samples were collected at predetermined time points (Table 1) and centrifuged at $15000 \mathrm{rpm}, 4^{\circ} \mathrm{C}$ for $10 \mathrm{~min}$, the resulting supernatant plasma was transferred to tubes and stored at $-30^{\circ} \mathrm{C}$ prior to analysis. The collected samples (lower eyelid skin, conjunctiva, and eyeball) were washed thrice with PBS, patted dry, and weighed. Tissue samples were added with acetonitrile $(500 \mu \mathrm{L})$ and mixed by means of a homogenizer (Polytron PT 1200 E; Kinematica AG, Littau, Lucerne, Switzerland) at $12000 \mathrm{rpm}, 4^{\circ} \mathrm{C}$ for $5 \mathrm{~min}$. The concentration of tranilast in plasma and tissues was quantified using liquid chromatography-tandem mass spectrometry (LC-MS/MS).

Quantification of Tranilast Using LC-MS/MS The tissue homogenates or plasma sample $(50 \mu \mathrm{L})$ were mixed with an equivalent amount of acetonitrile and centrifuged at $15000 \mathrm{rpm}, 4^{\circ} \mathrm{C}$ for $5 \mathrm{~min}$. The resulting supernatant $(10 \mu \mathrm{L})$ was injected into an LC-MS/MS system for the quantification of tranilast. The LC-MS/MS system consisted of a system controller (CBM-20 A; Shimadzu Corporation, Kyoto, Japan), pump (LC-20AD; Shimadzu Corporation), auto-sampler (SIL20ACHT; Shimadzu Corporation), column oven (CTO-20A; Shimadzu Corporation), detector (4000QTRAP; AB Sciex LLC, Tokyo Japan), and analysis software (Analyst ${ }^{\circledR}$ version 1.4.2; Shimadzu Corporation). The column (Shodex ODP2HPG-2A $2.0 \times 10 \mathrm{~mm}$, Showa Denko Inc., Tokyo, Japan) was kept at $40^{\circ} \mathrm{C}$. The mobile phase was a mixture of acetonitrile and $0.05 \%$ formic acid containing $5 \mathrm{mM}$ ammonium: acetate $(80: 20)$ with a flow rate $0.2 \mathrm{~mL} / \mathrm{min}$. Mass spectrometric quantification was carried out in the multiple reaction monitoring mode, monitoring transition ions of $\mathrm{m} / \mathrm{z} 328$ to $m / z$ 191. Calibration was carried out with six different concentrations within a range of $0.1-1000 \mathrm{ng} / \mathrm{mL}$ with a correlation coefficient of 0.99 .

Pharmacokinetic Calculation and Statistical Analysis The pharmacokinetic calculation presented in this section is based on previous studies. ${ }^{5,10)}$ A two-compartmental open model was used for intravenous administration and a noncompartmental analysis was applied for eyelid application. The main pharmacokinetic parameters for intravenous administration were derived from a graphical analysis of the concentration-time curve and subsequently used for the calculation of the corresponding secondary parameters. Experimental data with $p<0.05$ were considered statistically significant by means of one-way ANOVA and Tukey's honestly significant

Table 1. Design for Animal Studies

\begin{tabular}{lcccc}
\hline \hline Method of administration & $\begin{array}{c}\text { Dose } \\
(\mathrm{mg} / \mathrm{kg})\end{array}$ & $\begin{array}{c}\text { Drug } \\
\text { concentration } \\
(\% \mathrm{w} / \mathrm{v})\end{array}$ & $\begin{array}{c}\text { Volume } \\
\text { administered } \\
(\mu \mathrm{L})\end{array}$ & $\begin{array}{c}\text { Sampling time } \\
\text { points }(\mathrm{h})\end{array}$ \\
\hline $\begin{array}{c}\text { Topical application } \\
\text { (lower eyelid skin) }\end{array}$ & 0.5 & 0.1 & 100 & $\begin{array}{c}\text { Samples } \\
\text { collected }\end{array}$ \\
$\begin{array}{l}\text { Intravenous injection } \\
\text { (tail vein) } \\
\text { Eye drops }\end{array}$ & 0.5 & 0.1 & 100 & $\begin{array}{c}\text { Blood, lower eyelid skin, } \\
\text { conjunctiva, eyeball }\end{array}$ \\
\hline
\end{tabular}

*Maximum capacity of rat conjunctiva. Spillage of administered drug occurs beyond this volume.

A) Topical eyelid application

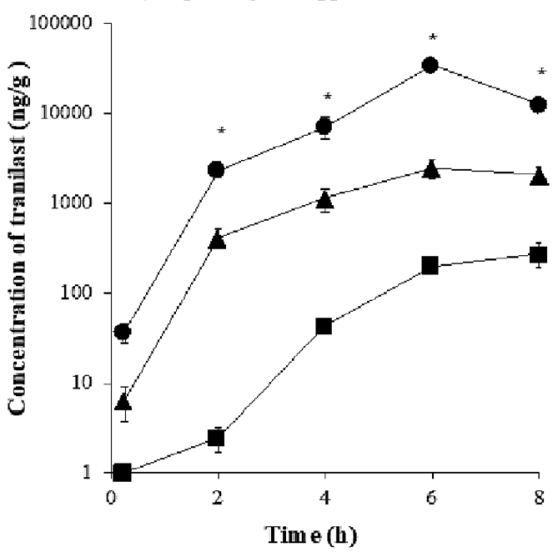

B) Eye drops

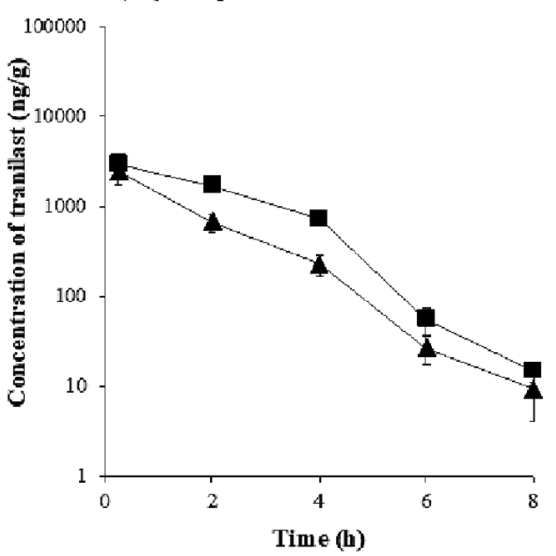

C) Intravenous

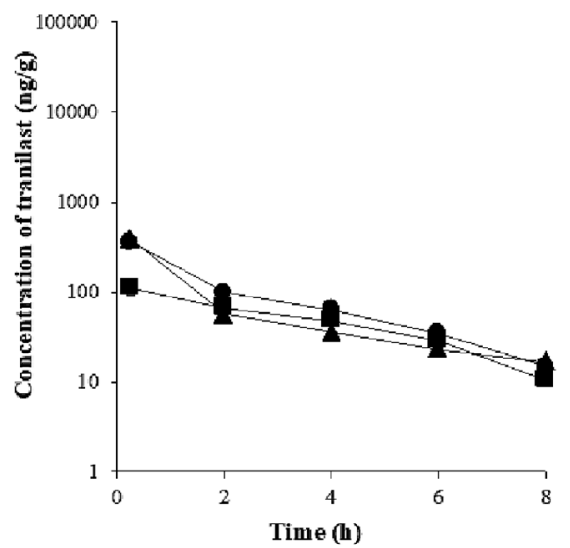

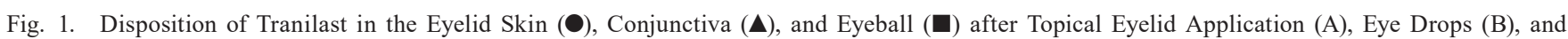
Intravenous Administration (C)

Each value represents the mean \pm standard deviation $(n=3)\left({ }^{*} p<0.05\right)$. 


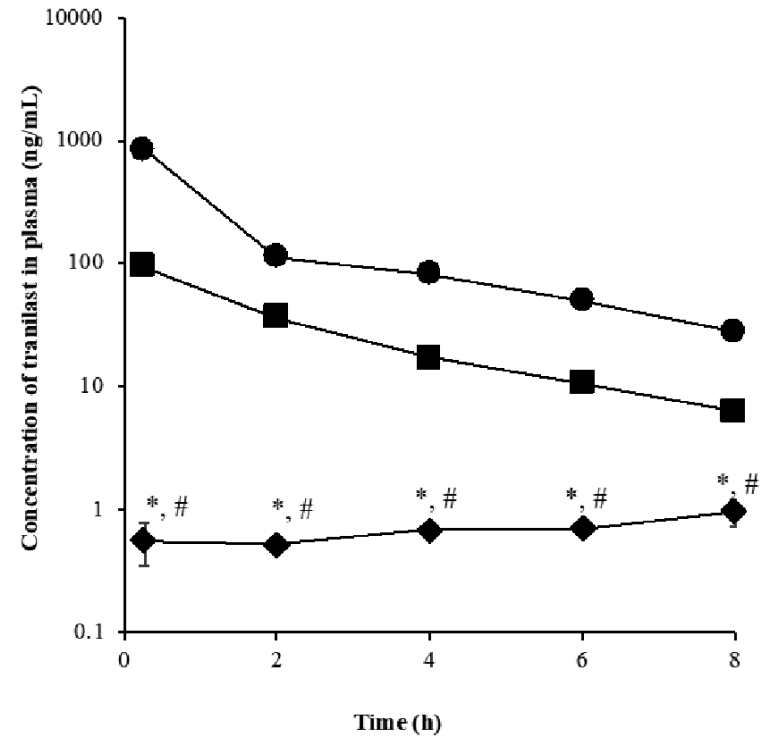

Fig. 2. Plasma Concentration-Time Profile of Tranilast after Topical Eyelid Application $(\bullet)$, Eye Drops $(\boldsymbol{\square})$, and Intravenous Administration (O)

Each value represents the mean \pm standard deviation $(n=3)$. Significant difference $(p<0.05)$ between topical eyelid application and *eye drops or *intravenous administration.

difference post hoc analysis. All data were expressed as mean with standard deviation ( \pm S.D.).

\section{Results}

Tissue Distribution of Tranilast Figure 1 shows the tranilast concentration in plasma and tissues following the three methods of application. Tranilast solution applied topically on the eyelids exhibited the highest concentration of $1.25 \times 10^{4} \mathrm{ng} / \mathrm{g}$ in the eyelid skin at $8 \mathrm{~h}$, followed by the conjunctiva and eyeball at 714 and $272 \mathrm{ng} / \mathrm{g}$, respectively. A distinctive sustained concentration-time profile of tranilast was observed for topical eyelid application.

Intravenous injection and eye drops revealed closely related concentration-time profiles but opposing to that of the topical application. Tranilast concentrations peaked $0.25 \mathrm{~h}$ (Figs. 1B, 1C) after administration and decreased quickly thereafter in a first-order fashion. At $8 \mathrm{~h}$ post eye drop administration, eyeball and conjunctiva concentrations were 14.8 and $9.44 \mathrm{ng} / \mathrm{g}$, respectively, and 10.4 and $16.5 \mathrm{ng} / \mathrm{g}$ for intravenous, correspondingly. Eye drops and intravenous administration exhibited 18- to 26-fold and 43- to 76-fold lower concentrations of tranilast in the eyeball and conjunctiva, respectively, compared with the topical eyelid application.

Plasma Concentration of Tranilast Figure 2 illustrates the typical plasma concentration-time profile of tranilast following the three routes of administration. The intravenous route and eye drops yielded a biphasic elimination of tranilast with visible distribution and elimination phases. High concentrations of tranilast $(854 \mathrm{ng} / \mathrm{mL})$ were detected in plasma after intravenous administration. In the case of topical eyelid application, $0.56-0.96 \mathrm{ng} / \mathrm{mL}$ tranilast was quantified in plasma suggesting low concentrations of tranilast in the systemic circulation. The concentration of tranilast in plasma were significantly different among the methods employed in the study, and topical eyelid application resulted in the lowest concentration of tranilast.
Table 2. Pharmacokinetic Parameters for a Two-Compartment Open Model after Intravenous Injection of Tranilast

\begin{tabular}{|c|c|c|c|}
\hline Parameters & & & $\begin{array}{c}\text { Dose } \\
0.5 \mathrm{mg} / \mathrm{kg}\end{array}$ \\
\hline $\begin{array}{l}\text { Hypothetical concentration at } \\
\alpha \text {-phase }\end{array}$ & $A$ & $\mathrm{ng} / \mathrm{mL}$ & $983 \pm 22$ \\
\hline $\begin{array}{l}\text { Hypothetical concentration at } \\
\beta \text {-phase }\end{array}$ & $B$ & $\mathrm{ng} / \mathrm{mL}$ & $241 \pm 17$ \\
\hline Concentration at time 0 & $C_{\mathrm{p}}^{0}$ & $\mathrm{ng} / \mathrm{mL}$ & $1224 \pm 13$ \\
\hline Slope of the distribution phase & $\alpha$ & $\mathrm{h}^{-1}$ & $1.79 \pm 0.09$ \\
\hline Slope of the elimination phase & $\beta$ & $\mathrm{h}^{-1}$ & $0.27 \pm 0.11$ \\
\hline Half-life time for distribution phase & $t_{0.5, \beta}$ & $\mathrm{h}$ & $2.58 \pm 0.18$ \\
\hline $\begin{array}{l}\text { Distribution rate constant from } \\
\text { tissue compartment }\end{array}$ & $k_{21}$ & $\mathrm{~h}^{-1}$ & $0.57 \pm 0.10$ \\
\hline $\begin{array}{l}\text { Distribution rate constant to } \\
\text { tissue compartment }\end{array}$ & $k_{12}$ & $\mathrm{~h}^{-1}$ & $0.64 \pm 0.04$ \\
\hline Elimination (overall) rate constant & $k_{10}$ & $\mathrm{~h}^{-1}$ & $0.85 \pm 0.12$ \\
\hline $\begin{array}{l}\text { Volume of distribution, } \\
\text { central compartment }\end{array}$ & $V_{\mathrm{c}}$ & $\mathrm{mL} / \mathrm{kg}$ & $408 \pm 132$ \\
\hline $\begin{array}{l}\text { Volume of distribution, } \\
\text { peripheral compartment (tissues) }\end{array}$ & $V_{\mathrm{T}}$ & $\mathrm{mL} / \mathrm{kg}$ & $464 \pm 63$ \\
\hline Area under the curve & $A U C$ & $\mathrm{ng} \cdot \mathrm{h} / \mathrm{mL}$ & $1449 \pm 119$ \\
\hline Clearance & $C L_{\text {tot }}$ & $\mathrm{mL} / \mathrm{h} / \mathrm{kg}$ & $345 \pm 148$ \\
\hline
\end{tabular}

Each value represents the mean \pm S.D. $(n=4)$.

Pharmacokinetics of Tranilast Figure $2(\mathbf{)})$ shows the plasma concentration-time profile after intravenous injection at $0.5 \mathrm{mg} / \mathrm{kg}$. Table 2 shows the corresponding pharmacokinetic parameters. The tranilast concentration in plasma declined with two distinctive elimination phases. Hence, a two-compartmental model was applied for the intravenous data shown in Table 2. In addition, Table 3 shows the noncompartmental pharmacokinetics parameters for plasma and tissues.

Eyelid skin application, eye drops, and intravenous routes have mean residence times (MRTs) of $8.07,1.79$, and $3.25 \mathrm{~h}$ in the eyeball and $10.8,1.29$, and $2.97 \mathrm{~h}$ in the conjunctiva, correspondingly (Table 3 ). In the eyeball, topical application of tranilast onto the eyelids corresponded to a 4.5- and 2.5-fold higher MRT compared with eye drops and intravenous administration, respectively. An 8.4- or 3.6-fold higher MRT was observed in the conjunctiva after topical application compared with eye drops or intravenous administration, respectively. This denote a slow penetration of tranilast into the ocular tissues, subsequently a slow elimination from these target tissues.

The relationship of MRT and tissue-to-plasma concentration ratio of tranilast is presented in Fig. 3. MRTs of tranilast and its tissue-to-plasma concentration exhibited a positive correlation, wherein a longer MRT corresponded to a higher tissueto-plasma concentration.

\section{Discussion}

In this study, we confirmed that topical application of a lipophilic model drug, tranilast, on the eyelid skin prolonged its distribution in the ocular tissues. Three methods of administration were employed to elucidate the effect of topical eyelid application of drugs using a pharmacokinetic approach. This is the first study to demonstrate the delivery of tranilast into 
Table 3. Mean Non-compartmental Pharmacokinetic Parameters of Tranilast in Rats after Intravenous Injection, Topical Eyelid, and Eye Drop Administration

\begin{tabular}{|c|c|c|c|c|c|c|c|c|c|c|c|}
\hline $\begin{array}{c}\text { Drug } \\
\text { application }\end{array}$ & Organ & $\begin{array}{c}A U C_{(0-t)} \\
(\mathrm{ng} \cdot \mathrm{h} / \mathrm{mL})\end{array}$ & $\begin{array}{l}A U C_{(0-\infty)} \\
(\mathrm{ng} \cdot \mathrm{h} / \mathrm{mL})\end{array}$ & $\begin{array}{l}A U M C_{(0-t)} \\
\left(\mathrm{ng} \cdot \mathrm{h}^{2} / \mathrm{mL}\right)\end{array}$ & $\begin{array}{l}A U M C_{(0-\infty)} \\
\left(\mathrm{ng} \cdot \mathrm{h}^{2} / \mathrm{mL}\right)\end{array}$ & $\lambda_{z}\left(\mathrm{~h}^{-1}\right)$ & $M R T(\mathrm{~h})$ & $M A T(\mathrm{~h})$ & $\begin{array}{c}V d_{\mathrm{ss}} \\
(\mathrm{mL} / \mathrm{kg})\end{array}$ & $K_{\mathrm{el}}\left(\mathrm{h}^{-1}\right)$ & $t_{0.5}(\mathrm{~h})$ \\
\hline \multirow[t]{4}{*}{ I.v. injection } & Plasma & 1357 & 1461 & 2108 & 3325 & 0.27 & 2.28 & $\mathrm{~N} / \mathrm{A}$ & 6235 & 0.44 & 1.58 \\
\hline & Eyelid skin & 754 & 794 & 1500 & 1939 & 0.36 & 2.44 & $\mathrm{~N} / \mathrm{A}$ & 12290 & 0.41 & 1.69 \\
\hline & Conjunctiva & 628 & 712 & 1000 & 2113 & 0.19 & 2.97 & N/A & 16650 & 0.34 & 2.06 \\
\hline & Eyeball & 398 & 425 & 1088 & 1380 & 0.38 & 3.25 & $\mathrm{~N} / \mathrm{A}$ & 30550 & 0.31 & 2.25 \\
\hline \multirow{4}{*}{$\begin{array}{l}\text { Topical } \\
\text { eyelid } \\
\text { application }\end{array}$} & Plasma & 5 & 16 & 24 & 238 & 0.09 & 14.6 & 12.3 & 3598000 & 0.07 & 10.2 \\
\hline & Eyelid skin & 99090 & 184700 & 574300 & 1846800 & 0.15 & 10.0 & 7.56 & 217 & 0.10 & 6.93 \\
\hline & Conjunctiva & 9923 & 23440 & 56030 & 254000 & 0.15 & 10.8 & 7.83 & 1849 & 0.09 & 7.51 \\
\hline & Eyeball & 758 & 1342 & 4898 & 10820 & 0.47 & 8.07 & 4.82 & 24050 & 0.12 & 5.59 \\
\hline \multirow[t]{4}{*}{ Eye drops } & Plasma & 222 & 247 & 472 & 774 & 0.25 & 3.13 & 0.85 & 50620 & 0.32 & 2.17 \\
\hline & Eyelid skin & \multicolumn{10}{|c|}{ Not determined } \\
\hline & Conjunctiva & 4247 & 4258 & 5371 & 5481 & 0.80 & 1.29 & -1.68 & 1209 & 0.77 & 0.89 \\
\hline & Eyeball & 7759 & 7774 & 13800 & 13940 & 0.97 & 1.79 & -1.46 & 922 & 0.56 & 1.24 \\
\hline
\end{tabular}

$A U C_{(0-t)}:$ area under plasma concentration-time curve; $A U C_{(0-\infty)}$ area under plasma concentration-time curve extrapolated to infinity; $A U M C_{(0-t)}:$ area under first moment curve; $A U M C_{(0-\infty)}$ : area under first moment curve extrapolated to infinity; $\lambda_{z}$ : terminal elimination rate constant; $M R T$ : mean residence time; $M A T$ : mean absorption time; $V d_{\mathrm{ss}}$ : apparent volume of distribution at steady state; $K_{\mathrm{el}}$ : elimination rate constants; $t_{0.5}$ : half-life.

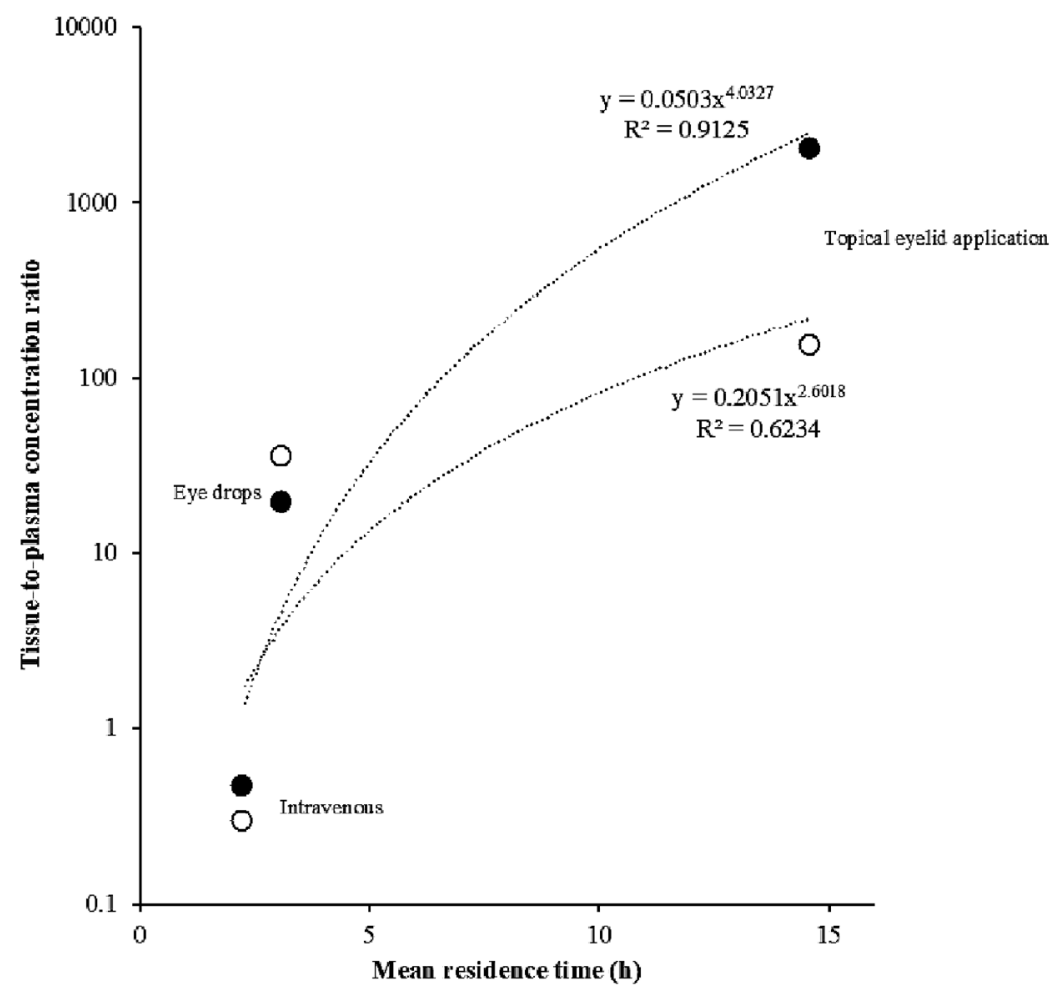

Fig. 3. The Correlation of Mean Residence Time with Tissue-to-Plasma Concentration Ratio of Tranilast in the Eyeball (O) and Conjunctiva

ocular tissues through the lower eyelids.

The concentration of tranilast decreased linearly in a first order fashion over an $8 \mathrm{~h}$ period for both eye drops and intravenous administration. The use of eye drops is viewed as easy and convenient, nevertheless eye drops are restricted in terms of pharmacologic effects. Several studies reported that the use of eye drops was effective in treating the surface of the eye only for a short time, hence its bioavailability was extremely low (i.e., 5-10\%). ${ }^{11,12)}$ In the present study, eye drops were unable to sustain the concentration of tranilast for $8 \mathrm{~h}$, which had profiles closely resembling intravenous administration. The rapid decline in tranilast in the ocular tissues after eye drop administration implies the need for repeated instillation of concentrated solutions to achieve the desired therapeutic efficacy in the anterior and posterior hemispheres of the eye. The use of eye drops in chronic ocular conditions (e.g., eye infections, macular degenerative disorders) warranting frequent instillation is impractical and promotes poor patient compli- 
ance. Moreover, high plasma concentrations of tranilast were obtained after eye drop and intravenous administration, which signifies extensive systemic circulation, an occurrence associated with the development of unwanted side effects.

Topically application of tranilast solution onto the lower eyelid skin exhibited sustained features, with the eyelid skin having the highest tranilast concentration followed by the conjunctiva and the eyeball. This finding implied a lower frequency of dosing and an anticipated longer duration of therapeutic effect in the ocular region. High concentrations of tranilast were expected in the eyelid skin, the application site. Our in vitro studies using tranilast solution showed that tranilast has an eyelid skin permeability coefficient of $(3.66 \pm 1.25) \times 10^{-7} \mathrm{~cm} / \mathrm{s}$ whereas the abdominal skin has a permeability coefficient of $(0.34 \pm 0.13) \times 10^{-7} \mathrm{~cm} / \mathrm{s}$ indicating an 11-fold higher permeation coefficient through the eyelid skin. The permeability coefficient of tranilast solution through the eyelid skin was at par with the transdermal drug delivery systems on the market. ${ }^{1)}$ The eyelid skin has only 8 layers of stratum corneum, which is thin enough to support drug penetration from the eyelids into ocular target regions, including the conjunctiva and eyeball. Our previous work using a hydrophilic model drug, pilocarpine, demonstrated a decrease in pupil size with passage of time following topical eyelid application, signifying sustained pharmacologic effect of a hydrophilic pilocarpine. ${ }^{5)}$

The mechanism governing the migration of tranilast into the eyes is initially by drug partitioning into the stratum corneum and transport by passive diffusion. It further penetrates the deeper layers of the stratum corneum and into the viable epidermis and dermis, the skin layer adjacent to the conjunctiva, and finally distributes into the eyeball. Therefore, the conjunctiva and eyeball exhibited detectable amounts of tranilast after topical administration on the eyelids.

Tranilast exhibited area under the curve (AUC) values of $9923 \mathrm{ng} \cdot \mathrm{h} / \mathrm{mL}$ in the conjunctiva after topical eyelid application, which corresponded to 2-fold higher concentration compared with eye drops. This result is valuable in the management of allergic conjunctivitis, where the condition afflicts predominantly the conjunctiva. Moreover, the conjunctiva can act as a reservoir for the sustained delivery of lipophilic molecules such as antibiotics and steroids. The mean residence times of tranilast following topical eyelid application was significantly higher by $2.5-4.5$-fold in the eyeball and 3.6-8.4-fold in the conjunctiva, suggesting a slower rate of absorption and subsequently slower elimination process. Consequently, this allows the drug to accumulate in the conjunctiva and eyeball for an extended duration and exhibit its prolonged pharmacologic effect. This demonstrates that tranilast stayed in a particular ocular compartment for a longer time when administered topically onto the eyelids. In addition, a 1.3-fold higher tranilast MRT was found in the conjunctiva and eyeball compared with the MRT of pilocarpine in the same target tissues based on our previous work. This finding may be attributed to the difference in lipophilicities wherein tranilast, as a lipophilic drug, exhibits higher binding capacity to the sclera and, therefore, longer transport lag time. ${ }^{13)}$

In the current study, the delivery of tranilast by the lower eyelid skin proved to be a superior method over eye drops and intravenous administration. This affirms the possibility of delivering tranilast through the eyelids for chronic eye diseases. This method of application is beneficial in allergic conjunctivitis and viral infections where there is the necessity to deliver a drug at a constant and effective concentration and maintained over a long period. Topical eyelid skin application will improve treatment effectiveness by delivering a drug during long sleeping time when the use of liquid and semi-solid eye preparations is deemed impossible. Subsequent studies should highlight the quantification of drugs in specific eye regions after topical eyelid application to understand deeper the migration of tranilast from the anterior to the posterior hemisphere of the eyes using large animal models or human skin. Delivery of high molecular weight drugs and large molecules through the eyelid skin must be investigated in future studies.

\section{Conclusion}

Topically applied tranilast solution onto the lower eyelids showed the highest mean residence times and high drug concentrations were quantified in the ocular tissue targets, conjunctiva and eyeball, indicating exclusive accumulation of the drug in the aforementioned ocular targets. Therefore, it is reasonable to prepare lipophilic drugs as pharmaceutical formulations aimed at the eyelids suitable for chronic treatment.

Conflict of Interest The authors declare no conflict of interest.

\section{References}

1) See G. L., Sagesaka A., Sugasawa S., Todo H., Sugibayashi K., Int. J. Pharm., 533, 198-205 (2017).

2) Sernicola A., Gattazzo I., Di Staso F., Giordano D., Capalbo A., Persechino F., Scuderi G., Pereschino S., Dermatol. Ther. 32, e13134 (2019)

3) Isowaki A., Ohtori A., Matsuo Y., Tojo K., Biol. Pharm. Bull., 26, 69-72 (2003).

4) See G. L., Sagesaka A., Torizuka H., Todo H., Sugibayashi K. J. Drug Deliv. Sci. Technol., 47, 380-385 (2018).

5) See G. L., Sagesaka A., Todo H., Wierzba K., Sugibayashi K., J. Pharm. Sci., 108, 2942-2948 (2019).

6) Itoh F., Komatsu Y., Taya F., Isaji M., Kojima M., Momose Y., Suzawa H., Miyata H., Shibazaki T., Nippon Yakurigaku Zasshi, 101, 27-32 (1993)

7) Choi S. U., Kim K. W., Moon N. J., BMC Ophthalmol., 16, 1-7 (2016).

8) Adachi T., Fukuda K., Kondo Y., Nishida T., Invest. Ophthalmol. Vis. Sci., 51, 3954-3960 (2010).

9) Tobari I., Iwaki Y., Miyake K., J. Cataract Refract. Surg., 25, 1394-1399 (1999).

10) Rosenbaum S., "Pharmacokinetics of an intravenous bolus injection in a two-compartment model," John Wiley \& Sons Inc., New Jersey, 2011.

11) Baranowski P., Karolewicz B., Gajda M., Pluta J., ScientificWorldJournal, 2014, 861904 (2014).

12) Agrahari V., Mandal A., Agrahari V., Trinh H. M., Joseph M., Ray A., Hadji H., Mitra R., Pal D., Mitra A. K., Deliv. Transl. Res., 6, 735-754 (2016).

13) Wen H., Hao J., Li S. K., Pharm. Res., 27, 2446-2456 (2011). 